ARTICLE

Received 29 Apr 2014 | Accepted 3 Oct 2014 | Published 17 Nov $2014 \quad$ DOl: 10.1038/ncomms6473

\title{
FCA mediates thermal adaptation of stem growth by attenuating auxin action in Arabidopsis
}

Hyo-Jun Lee ${ }^{1}$, Jae-Hoon Jung ${ }^{1}$, Lucas Cortés Llorca ${ }^{2}$, Sang-Gyu Kim² ${ }^{2}$ Sangmin Lee ${ }^{1}$, Ian T. Baldwin ${ }^{2} \&$ Chung-Mo Park ${ }^{1,3}$

Global warming is predicted to profoundly affect plant distribution and crop yield in the near future. Higher ambient temperature can influence diverse aspects of plant growth and development. In Arabidopsis, the basic helix-loop-helix transcription factor PHYTOCHROMEINTERACTING FACTOR 4 (PIF4) regulates temperature-induced adaptive responses by modulating auxin biosynthesis. At high temperature, PIF4 directly activates expression of YUCCA8 (YUC8), a gene encoding an auxin biosynthetic enzyme, resulting in auxin accumulation. Here we demonstrate that the RNA-binding protein FCA attenuates PIF4 activity by inducing its dissociation from the YUC8 promoter at high temperature. At $28^{\circ} \mathrm{C}$, auxin content is elevated in FCA-deficient mutants that exhibit elongated stems but reduced in FCAoverexpressing plants that exhibit reduced stem growth. We propose that the FCA-mediated regulation of YUC8 expression tunes down PIF4-induced architectural changes to achieve thermal adaptation of stem growth at high ambient temperature.

\footnotetext{
${ }^{1}$ Department of Chemistry, Seoul National University, Seoul 151-742, Korea. ${ }^{2}$ Department of Molecular Ecology, Max Planck Institute for Chemical Ecology, 07745 Jena, Germany. ${ }^{3}$ Plant Genomics and Breeding Institute, Seoul National University, Seoul 151-742, Korea. Correspondence and requests for materials should be addressed to C.-M.P. (email: cmpark@snu.ac.kr).
} 
P lants adapt to high ambient temperature by adjusting plant architecture, such as elongation of hypocotyl and leaf stem and increase of leaf hyponasty ${ }^{1}$. These architectural adaptations help plants minimize the chance of heat damage and enhance evaporative leaf cooling for optimized plant growth ${ }^{2}$. However, a trade-off occurs when plants obtain the fitness benefits during long-term exposure to high temperature: plants having abnormally elongated stems tend to fall down and suffer from physiological imbalance ${ }^{3}$. This necessitates that there should be a fine-tuning mechanism that attenuates the effects of high temperature on stem growth.

The Arabidopsis basic helix-loop-helix transcription factor PHYTOCHROME-INTERACTING FACTOR 4 (PIF4) plays a major role in high-temperature-induced plant responses. Elongation of hypocotyl, increase of hyponasty and induction of flowering are suppressed by pif4 mutations under high temperature conditions $^{1,4,5}$. It has been reported that PIF4 directly regulates transcription of auxin biosynthesis enzyme genes, such as YUCCA8 (YUC8), resulting in accumulation of auxin content ${ }^{5}$. PIF4 function is enhanced at high temperature. The PIF4 transcription is induced by exposure to high temperature ${ }^{5,6}$. In addition, protein stability and DNA-binding affinity of PIF4 are elevated at high ambient temperatures ${ }^{6,7}$. While the role of PIF4 in thermal regulation of stem growth is evident, it is not fully understood how PIF4 function is modulated at the molecular level.

The Arabidopsis RNA-binding protein FCA is a critical component of the autonomous flowering pathway, which induces flowering by suppressing the floral repressor FLOWERING LOCUS $C(F L C)^{8,9}$. FCA plays a role in alternative polyadenylation of its own pre-mRNA and antisense FLC RNA ${ }^{8,10}$. FCA also acts as a chromatin remodelling factor: it mediates histone demethylation of FLC chromatin, causing suppression of FLC expression ${ }^{9}$. Notably, it has been reported that FCA incorporates ambient temperature signals into the thermosensory flowering pathway ${ }^{11}$, raising a possibility that FCA would participate in plant responses to changes in ambient temperature.

In this work, we demonstrate that FCA mediates the dissociation of PIF4 from its target gene chromatin, such as YUC8, at high ambient temperature. FCA is recruited to YUC8 promoter through interaction with PIF4, resulting in the dissociation of PIF4 from YUC8 promoter and the suppression of YUC8 transcription. These observations illustrate that FCA attenuates PIF4 action in auxin accumulation occurring during high-temperature-induced stem growth to maintain architectural and physiological balance.

\section{Results}

FCA mediates thermal acceleration of stem growth. While working on the role of FCA in thermosensory flowering, we found that FCA-deficient mutants $f c a-9$ and $f_{c a}-11$ exhibited elongated hypocotyls at $28^{\circ} \mathrm{C}$ compared with those of wild-type (Col-0) plants (Fig. 1a). In contrast, FCA-overexpressing transgenic plants (35S:FCA) had shorter hypocotyls under identical conditions, suggesting that FCA is associated with ambient temperature regulation of hypocotyl growth.

We also examined the effects of $f c a$ mutation on leaf hyponasty and leaf stem growth, which are also affected by high temperature $^{1,12}$. Leaf hyponasty markedly increased in $f_{c a} a$ when grown at $28^{\circ} \mathrm{C}$, but it was reduced in 35S:FCA transgenic plants (Fig. 1b). In addition, leaf stem (petiole) was elongated in $f(a-9$ compared with that of Col-0 plants, but the high temperature sensitivity disappeared in 35S:FCA transgenic plants (Fig. 1c). These observations support the notion that FCA mediates the high ambient temperature-induced architectural modifications.

FCA-mediated ambient temperature signals regulate flowering time via the floral repressor $F L C^{8,9,11}$. To examine whether FLC is involved in the enhanced stem growth in $f c a-9$ at high temperature, we measured hypocotyl growth in FLC-deficient $f l c-3$ mutant and FLC-accumulating plants, such as $f l k-1$ and $f l d-6$ mutants $^{13,14}$, active FRIGIDA (FRI) allele-containing FRI-Col plants $^{15}$ and FLC-overexpressing plants. Their hypocotyl growth was similar to that of Col-0 plants at $28^{\circ} \mathrm{C}$ (Fig. 1d, Supplementary Fig. 1), showing that the accelerated hypocotyl elongation in $\mathrm{fca}-9$ at $28^{\circ} \mathrm{C}$ is not related with FLC.

IAA content is elevated in $f \boldsymbol{c a}-9$ at $28^{\circ} \mathrm{C}$. To obtain clues as to how FCA regulates stem growth at high temperature, we investigated the expression of genes encoding biosynthetic enzymes of ethylene, gibberellic acid, brassinosteriod and auxin, which mediate stem growth ${ }^{16,17}$. Among those, only auxin biosynthetic enzyme genes were significantly induced in $f c a-9$ when grown at $28^{\circ} \mathrm{C}$ (Supplementary Fig. 2). Among the INDOLE-3-ACETIC ACID INDUCIBLE (IAA) and YUC genes tested, IAA29 and YUC8, which have been shown to mediate high-temperatureinduced stem elongation ${ }^{1,5}$, were induced by more than sixfold in the high-temperature-treated $f c a-9$ mutant (Fig. 2a,b). Auxinresponsive SMALL AUXIN UPREGULATED 20 (SAUR20) and SAUR22 genes are also involved in high-temperature regulation of hypocotyl growth ${ }^{4}$. We found that they were expressed at higher levels in $f \mathrm{ca}-9$ compared with that in Col-0 plants at $23^{\circ} \mathrm{C}$ (Fig. 2c), and their transcripts levels were further elevated in the high-temperature-treated $f c a-9$ mutant.

The upregulation of IAA29, YUC8 and SAUR genes in $f c a-9$ at $28^{\circ} \mathrm{C}$ suggested that endogenous auxin content is elevated in $f_{c a}$ 9. To examine whether the elongated hypocotyl of $f c a-9$ at $28^{\circ} \mathrm{C}$ is caused by elevated auxin content, the mutant was grown at either 23 or $28^{\circ} \mathrm{C}$ in the presence of the auxin transport inhibitor $1-N$-naphthylphthalamic acid ${ }^{18}$. The high-temperature-induced hypocotyl elongation of the mutant was completely suppressed by 1-N-naphthylphthalamic acid (Fig. 2d). Direct measurements of IAA content revealed that at $28^{\circ} \mathrm{C}$, the level of free IAA was higher by $36 \%$ in $f(a-9$ but lower by $28 \%$ in $35 S$ :FCA transgenic plants compared with those in Col-0 plant (Fig. 2e), indicating that the high-temperature-induced hypocotyl elongation is due to elevated IAA content in $f c a-9$.

FCA interacts with PIF4 in thermal induction of stem growth. IAA29, SAUR20, SAUR22 and YUC8 genes are regulated by PIF4 at a high temperature ${ }^{1,4,5}$, suggesting that PIF4 plays a role in the induction of the auxin-responsive genes in $f_{c a}-9$. We first examined whether FCA interacts with PIF4. Bimolecular fluorescence complementation (BiFC) assays showed that they interact with each other in the nucleus (Fig. 3a, Supplementary Fig. 3). in vitro pull-down assays also supported the physical association between FCA and PIF4 (Fig. 3b).

We next explored the functional relationship between FCA and PIF4. A PIF4-deficient pif4-101 mutant was crossed with $f(a-9$, resulting $f c a-9$ pif4-101. The high-temperature-induced hypocotyl elongation phenotype of $f_{c a}-9$ was suppressed in $f_{c a}-9$ pif4-101, which is similar to the hypocotyl phenotype of pif4-101 (Fig. 3c, Supplementary Fig. 4). In addition, the inductive effects of high temperature on YUC8 transcription were not observed in $f_{c a}-9$ pif4-101 and pif4-101 (Fig. 3d). Together with the elevated IAA content in $f(a-9$, these observations indicate that FCA is associated with the PIF4-regulated auxin biosynthesis.

FCA regulates PIF4 binding to YUC8 chromatin. A question was how FCA regulates PIF4 function. PIF4 gene is induced when plants grown at $16^{\circ} \mathrm{C}$ are exposed to $28^{\circ} \mathrm{C}$ (ref. 6). We examined whether the high-temperature induction of PIF4 gene is regulated by FCA. The inductive effects of high temperature on PIF4 
a

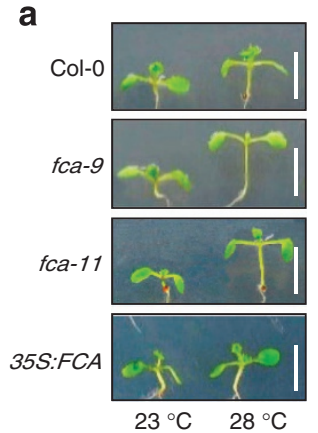

d

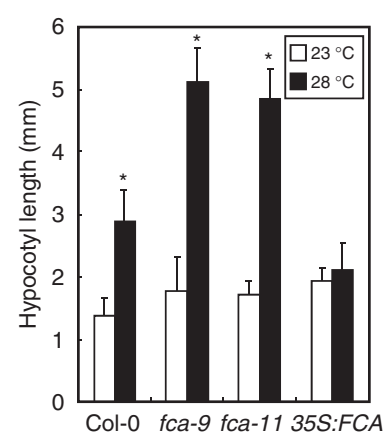

b
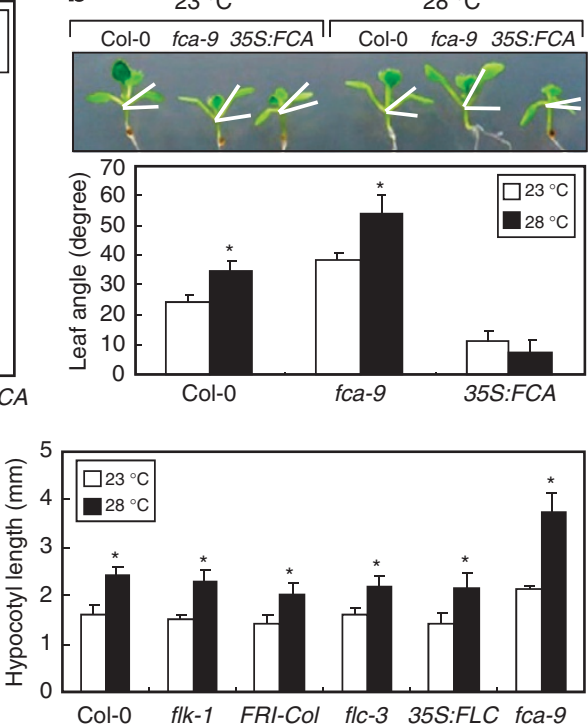

C
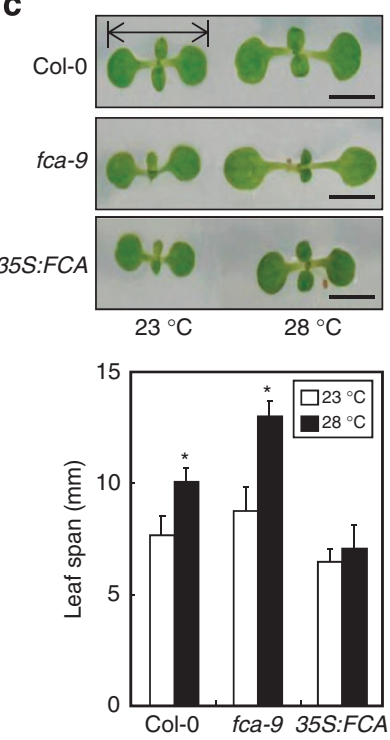

Figure 1 | FCA mediates the thermal acceleration of stem growth. Plants were grown on MS-agar plates at $23^{\circ} \mathrm{C}$ under continuous light conditions for 4 days and further grown at either 23 or $28^{\circ} \mathrm{C}$ for 3 days. Measurements of $15-20$ seedlings were averaged and statistically treated using Student $t$-test $\left({ }^{\star} P<0.01\right)$. Bars indicate s.e. (a) $f c a$ mutants have elongated hypocotyls at $28^{\circ} \mathrm{C}$. $(n=20)$. Scale bars, $1 \mathrm{~cm}$. (b) Hyponasty increases in $f c a-9$ at $28^{\circ} \mathrm{C}$. $(n=15)$. (c) Leaf span is elongated in $f c a-9$ at $28^{\circ} \mathrm{C}$. $(n=20)$. Scale bars, $5 \mathrm{~mm}$. (d) High-temperature-induced hypocotyl elongation of $f c a-9$ is not related with FLC. $(n=17)$.
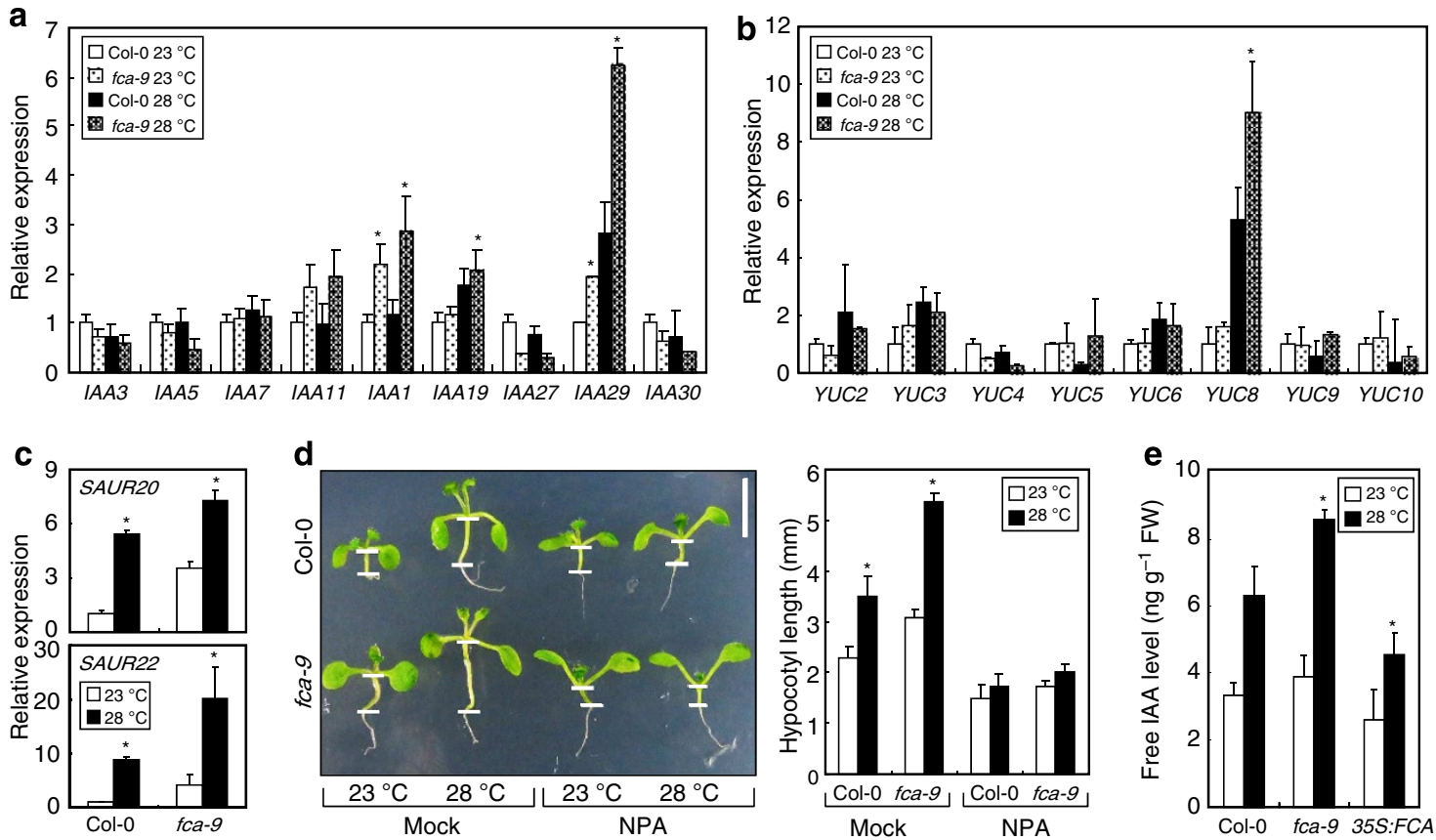

Figure 2 | IAA content is elevated in fca-9 at $28^{\circ} \mathbf{C}$. Transcript levels were measured by RT-qPCR. Biological triplicates were averaged and statistically treated using Student's $t$-test $\left({ }^{\star} P<0.01\right)$. Bars indicate s.e. (a) Transcript levels of IAA genes in fca-9. Plants were grown on MS-agar plates for 6 days at $23^{\circ} \mathrm{C}$ and exposed to $28^{\circ} \mathrm{C}$ for $24 \mathrm{~h}$ before harvesting whole plant materials. (b) Transcript levels of YUC genes in fca-9. Total RNA samples prepared in a were used for RT-qPCR. (c) Transcript levels of SAUR genes in fca-9. Total RNA samples prepared in a were used for RT-qPCR. (d) Elongated hypocotyls of fca-9 is compromised in the presence of $100 \mu \mathrm{M} 1-\mathrm{N}$-naphthylphthalamic acid (NPA). Plants were grown as described in Fig. 1 but in the presence of the auxin transport inhibitor NPA (left panel). Measurements of 20 hypocotyls were averaged and statistically treated using Student's $t$-test $\left({ }^{\star} P<0.01\right.$; right panel). (e) Measurements of free IAA content in fca-9. Plants were grown as described in a.

transcription were still evident in fca-9 (Supplementary Fig. 5). In addition, $f c a$ mutation did not affect the protein stability of PIF4 (Supplementary Fig. 6). We next examined whether $f c a$ mutation influences the transcriptional activity of PIF4 using a $\beta$-glucuronidase (GUS) transient expression system in Arabidopsis protoplasts ${ }^{19}$, which were prepared from $f c a-9$ mutant. It was found 
a

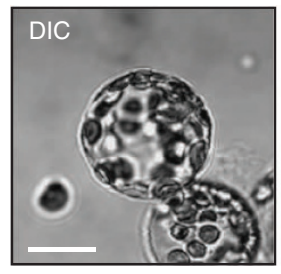

nYFP-PIF4/FCA-cYFP
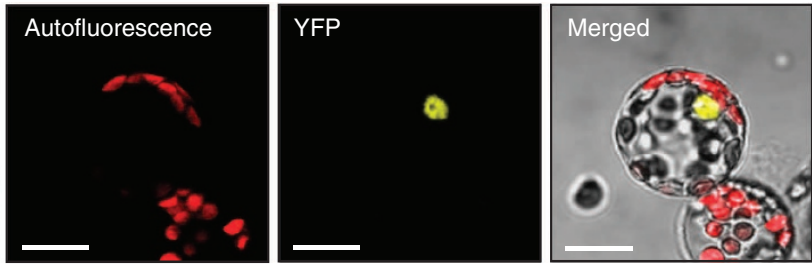

b
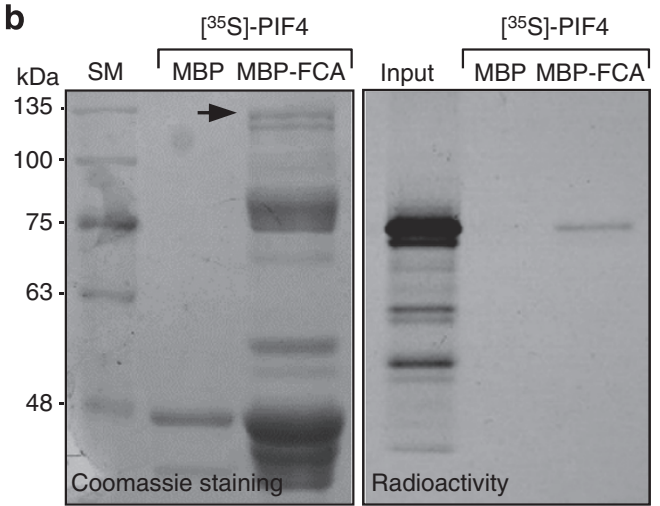

C

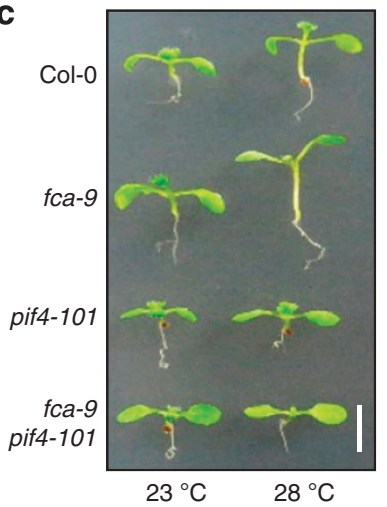

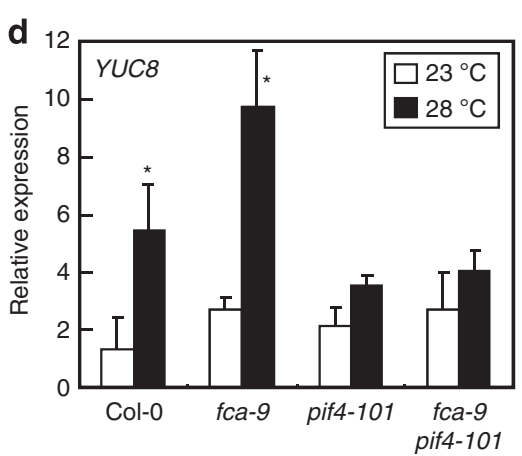

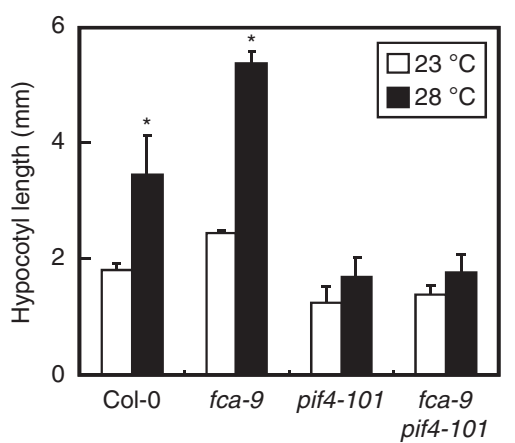

Figure 3 | FCA interacts with PIF4. (a) BiFC assays on FCA-PIF4 interaction. nYFP-PIF4 and cYFP-FCA gene fusions were coexpressed transiently in Arabidopsis protoplasts. FCA-PIF4 interactions were visualized by differential interference contrast microscopy. Scale bars, $10 \mu \mathrm{m}$. (b) In vitro pull-down assays on FCA-PIF4 interaction. Recombinant MBP-FCA fusion protein was produced in E. coli cells. ${ }^{35} \mathrm{~S}$-labelled PIF4 protein was prepared by in vitro translation. MBP alone was used as negative control (right panel). Part of Coomassie blue-stained gel was shown as loading control (left panel).

(c) Hypocotyl growth of fca-9 pif4-101. Plants grown on MS-agar plates for 4 days at $23^{\circ} \mathrm{C}$ were further grown at $28^{\circ} \mathrm{C}$ for 3 days (upper panel). Scale bar, $0.5 \mathrm{~cm}$. Measurements of 15 hypocotyls were averaged and statistically treated using Student's $t$-test $\left({ }^{\star} P<0.01\right.$; lower panel). Bars indicate s.e.

(d) Transcript levels of YUC8 gene in fca-9 pif4-101. Transcript levels were measured as described in Fig. 2. Biological triplicates were averaged and statistically treated using Student's $t$-test $\left({ }^{\star} P<0.01\right)$. Bars indicate s.e.

that the transcriptional activity of PIF4 was not altered in $f c a-9$, and coexpression of FCA did not affect the PIF4 activity (Supplementary Fig. 7), indicating that FCA does not affect the transcriptional activity of PIF4. Instead, it was hypothesized that FCA might influence the DNA binding of PIF4.

It has been known that PIF4 binds to G-box elements in YUC8 promoter, although it is unclear whether high temperature influences the DNA binding of PIF4 (ref. 5). Notably, it has recently been reported that PIF4 binds to FLOWERING LOCUS T (FT) promoter and its DNA binding is enhanced at high temperature $^{6}$. We therefore hypothesized that FCA might influence the binding of PIF4 to YUC8 promoter in response to high temperature. Chromatin immunoprecipitation (ChIP) assays revealed that the binding of PIF4 to a sequence region covering G-box elements in YUC8 promoter, which was designated P2 region in this work (Fig. 4a), was elevated in both Col-0 and $f_{c a-9}$ plants exposed to $28^{\circ} \mathrm{C}$ for $6 \mathrm{~h}$ (Fig. $4 \mathrm{~b}$ ). We next performed ChIP assays using plants that were exposed to $28^{\circ} \mathrm{C}$ for longer time (24 h). Notably, while the binding of PIF4 to P2 was reduced to a basal level in Col-0 plants, the high-level binding of PIF4 to P2 was maintained in $\mathrm{fca}_{-9}$ (Fig. 4c). Consistent with these observations, the transcript levels of YUC8 were similar in Col-0 and $f \mathrm{ca}-9$ that were exposed to $28^{\circ} \mathrm{C}$ for $6 \mathrm{~h}$ but was higher in $f_{c a}$ 9 than in Col- 0 when the plants were exposed to $28^{\circ} \mathrm{C}$ for $24 \mathrm{~h}$ (Fig. 4d). It was therefore concluded that FCA does not affect the binding step of PIF4 to YUC8 promoter but trigger the dissociation of PIF4 from YUC8 promoter, especially under long-term exposure to high temperature.

FCA induces chromatin modification at YUC8. FCA requires a histone demethylase, which targets the active marker histone-3 lysine-4 dimethylation (H3K4me2), to downregulate FLC expression $^{9}$. We therefore suspected that FCA influences the dissociation of PIF4 from YUC8 promoter via chromatin remodelling. ChIP assays showed that FCA binds to $\mathrm{P} 2$ region 
a

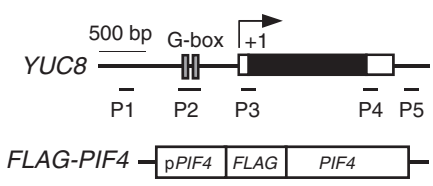

b
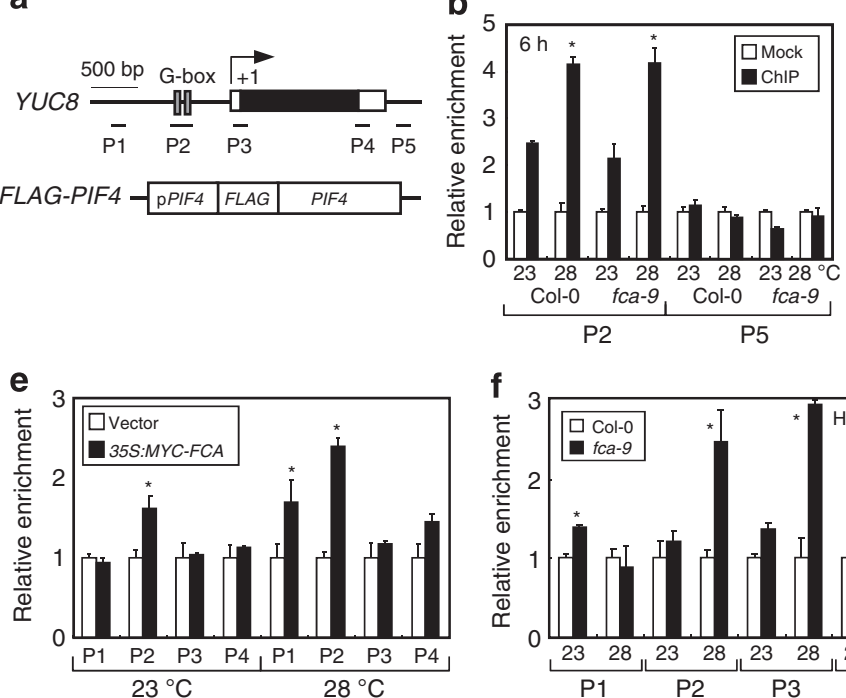

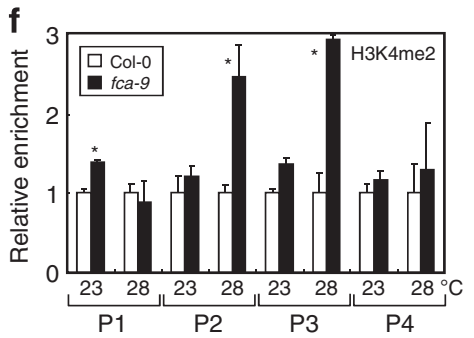

C
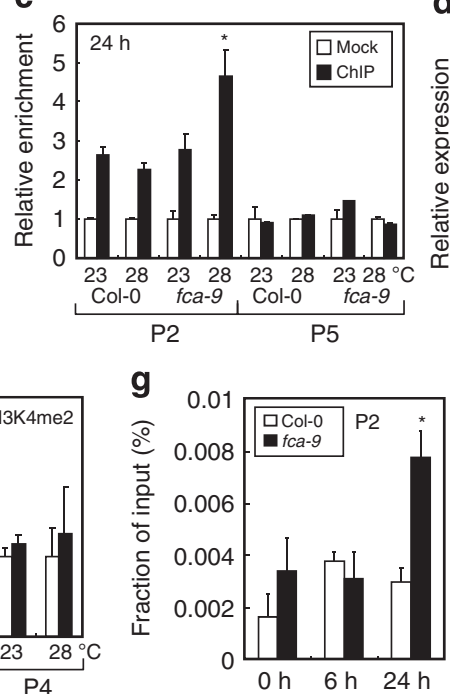
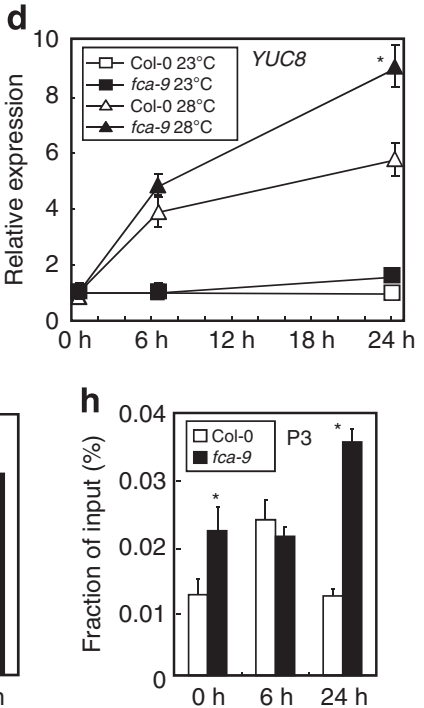

i

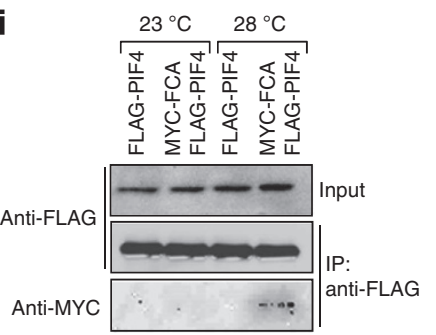

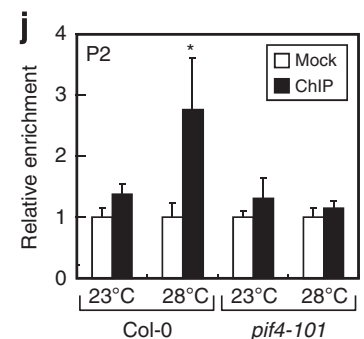

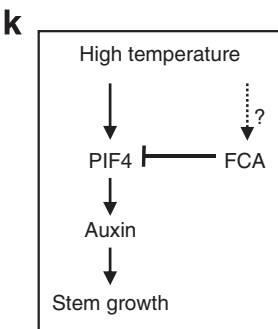

Figure 4 | FCA regulates PIF4 binding to YUC8 promoter. Biological triplicates were averaged and statistically treated using Student's $t$-test ( $P<0.01$ ). Bars indicate s.e. (a) Genomic structure of YUC8 gene. P1 to P5 sequence regions were used in ChIP assays. Black boxes are exons, and white boxes are untranslated regions. The FLAG-PIF4 expression construct, in which a FLAG octapolypeptide-coding sequence is fused in-frame to the $5^{\prime}$ end of PIF4 gene under the control of the endogenous PIF4 promoter consisting of approximately 2-kbp sequence upstream of the translation start site, is shown at the bottom. (b,c) ChIP assays on the effects of FCA on PIF4 binding to YUC8 promoter. The FLAG-PIF4 gene fusion was transformed into Col-0 and fca-9 plants. Plants grown on MS-agar plates for 6 days at $23^{\circ} \mathrm{C}$ were further grown at either 23 or $28^{\circ} \mathrm{C}$ for $6 \mathrm{~h}$ (b) and $24 \mathrm{~h}$ (c) before extracting chromatin from whole plants. ChIP assays were performed with P2 and P5 sequences using an anti-FLAG antibody. (d) Accumulation kinetics of YUC8 transcripts in fca- 9 after $28^{\circ} \mathrm{C}$ treatment. Transcript levels were measured as described in Fig. 2. (e) ChIP assays on FCA binding to YUC8 chromatin. A MYC-FCA fusion was overexpressed in Col-0 plants. Chromatin was prepared as described in c. ChIP assays were performed with P1 to P4 sequences using an anti-MYC antibody. (f) Histone modifications at YUC8 chromatin in fca-9. Plant growth and chromatin preparation were performed as described in c. ChIP assays were performed using an anti-H3K4me2 antibody. $(\mathbf{g}, \mathbf{h})$ Kinetics of histone modifications at YUC 8 chromatin in fca-9. Chromatin was prepared from plants at different time points after exposure to $28^{\circ} \mathrm{C}$. ChIP assays were performed with either P2 (g) or P3 (h) sequence using an anti-H3K4me2 antibody. (i) Coimmunoprecipitation assays on FCA-PIF4 interaction. The FLAG-PIF4 expression construct was transformed into 35S:MYC-FCA transgenic plants. Plants were treated with $28^{\circ} \mathrm{C}$, as described in c. Imunoprecipitation (IP) was performed using an anti-FLAG antibody. Input represents $5 \%$ of the IP reaction. (j) ChIP assays on FCA binding to YUC8 chromatin in pif4-101. The assays were performed as described in (c) using pif4-101 mutant overexpressing MYC-FCA driven by the CaMV 35 S promoter. (k) FCA attenuation of PIF4 action in thermal acceleration of stem growth. FCA regulates the binding of PIF4 to YUC8 promoter, providing an adaptation strategy at high temperature.

in YUC8 chromatin at $23^{\circ} \mathrm{C}$ and its DNA binding was considerably elevated at $28^{\circ} \mathrm{C}$ (Fig. 4e). FCA also bound to P1 region, which is approximately 500 bases upstream of $\mathrm{P} 2$ region, at $28^{\circ} \mathrm{C}$. In addition, the levels of $\mathrm{H} 3 \mathrm{~K} 4 \mathrm{me} 2$ was elevated by more than twofold in $\mathrm{P} 2$ region in $f c a-9$ at $28^{\circ} \mathrm{C}$ (Fig. 4f). The H3K4me2 level was also elevated in P3 region covering the transcription start site. These data suggest that FCA promotes the dissociation of PIF4 from the YUC8 promoter at $28^{\circ} \mathrm{C}$ by modifying YUC8 chromatin.

We next monitored the kinetics of $\mathrm{H} 3 \mathrm{~K} 4 \mathrm{me} 2$ accumulation in fca-9 after exposure to $28^{\circ} \mathrm{C}$. In Col-0, the H3K4me2 level in the $\mathrm{P} 2$ region was elevated by approximately twofold at $6 \mathrm{~h}$ but reduced to a basal level at $24 \mathrm{~h}$ (Fig. $4 \mathrm{~g}$ ). In $f c a-9$, it was largely unchanged at $6 \mathrm{~h}$ but significantly elevated by more than twofold at $24 \mathrm{~h}$. The kinetic patterns of H3K4me2 accumulation in P3 regions were similar to those observed in $\mathrm{P} 2$ regions of both Col-0 and $f_{c a-9}$ plants (Fig. 4h). We propose that FCA-mediated demethylation events are induced by long periods of exposure to high temperature.

FCA modulates the dissociation of PIF4 from YUC8 chromatin. A critical question was how high-temperature signals are incorporated into the FCA-PIF4-YUC8 module. FCA transcription was only moderately induced by approximately twofold at $28^{\circ} \mathrm{C}$ (Supplementary Fig. 8a). In addition, high temperature did not discernibly affect the FCA protein stability (Supplementary Fig. 8b). We next performed coimmunoprecipitation assays on Col-0 plants coexpressing FLAG-PIF4 and MYC-FCA fusions using anti-FLAG and anti-MYC antibodies for the pull down of the fusion proteins. FCA interacted with PIF4 in planta primarily at $28^{\circ} \mathrm{C}$ (Fig. 4i, Supplementary Fig. 9). Furthermore, ChIP assays 
on pif4-101 mutant overexpressing MYC-FCA showed that FCA binding to YUC8 chromatin did not occur in the mutant at $28^{\circ} \mathrm{C}$ (Fig. 4j). Together with the increased binding of PIF4 to YUC8 chromatin (Fig. 4b), these observations indicate that at high temperatures, PIF4 recruits FCA to the chromatin, where FCA induces chromatin modifications, causing PIF4 dissociation (Supplementary Fig. 10). All together, we conclude that FCA tunes down the high-temperature-induced stem elongation by attenuating PIF4 action probably through remodelling of YUC8 chromatin (Fig. 4k).

\section{Discussion}

We found that FCA, which is otherwise a well-characterized RNA-binding protein functioning in flowering time control ${ }^{8,9}$, plays a role in modulating PIF4 induction of YUC8 in response to high temperature. On the basis of our data, we propose that the FCA-mediated counteracting mechanism is required to offset the accelerated stem elongation at high temperature within the ambient temperature range, contributing to thermal adaptation of stem growth. The physiological role of FCA in this high temperature signalling is similar to that proposed for $\mathrm{HIGH}$ EXPRESSION OF OSMOTICALLY RESPONSIVE GENES 1 (HOS1) E3 ubiquitin ligase. In cold acclimation signalling, HOS1 triggers protein degradation of INDUCER OF CBF EXPRESSION 1 , which induces transcription of C-REPEAT BINDING FACTOR 1 and 3 (refs 20,21). The HOS1-mediated negative regulation of cold signalling is important for maintaining physiological balance under prolonged cold temperature conditions, because overinduction of cold response causes developmental defects and growth retardation ${ }^{22}$.

It is notable that FLOWERING LOCUS D (FLD), which acts together with FCA to modify FLC chromatin ${ }^{9}$, is not functionally related with FCA in thermal regulation of hypocotyl growth. While our data strongly support the role of FCA in YUC8 chromatin modification at high temperature, the observed modification of YUC8 chromatin in fca-9 background would simply reflect the thermal induction of YUC8 in the mutant. Future work will be required to clarify this uncertainty concerning the molecular mechanism of FCA in the transcriptional control of YUC8 and its functional relevance in thermal regulation of hypocotyl growth.

\section{Methods}

Plant materials and growth conditions. All Arabidopsis thaliana lines used were in Columbia (Col-0) background. Plants were grown in a controlled culture room at $23{ }^{\circ} \mathrm{C}$ with relative humidity of $55 \%$ under continuous light conditions, unless specified otherwise, with white light illumination $\left(120 \mu \mathrm{mol}\right.$ photons $\left.\mathrm{m}^{-2} \mathrm{~s}^{-1}\right)$ provided by fluorescent FLR40D/A tubes (Osram, Seoul, Korea). The $f c a-9, f c a-11$ and pif4-101 mutants and 35S:FCA transgenic plants have been described previously $1,23,24$. To generate 35S:MYC-FCA transgenic plants, a full-size FCA $\gamma$ cDNA was fused in-frame to the $3^{\prime}$ end of the MYC-coding sequence in the myc-pBA vector $^{25}$. The expression construct was transformed into Col-0 plants.

To examine the effects of high temperature on hypocotyl growth, leaf spanning and leaf hyponasty, plants grown on $1 / 2 \times$ Murashige and Skoog-agar plates (MSagar plates) at $23^{\circ} \mathrm{C}$ for 4 days were further grown at either 23 or $28^{\circ} \mathrm{C}$ for 3 additional days. Plants (15-20) were measured and averaged. To analyse the effects of high temperature on gene expression, plants grown at $23^{\circ} \mathrm{C}$ for 6 days on MSagar plates were further grown at either 23 or $28^{\circ} \mathrm{C}$ for $24 \mathrm{~h}$. Whole plants were used for the extraction of total RNA.

\footnotetext{
Analysis of gene transcript levels. Transcript levels were determined by reverse transcription-mediated quantitative real-time PCR (RT-qPCR). RT-qPCR reactions were carried out according to the guidelines that have been proposed to guarantee reproducible and accurate measurements ${ }^{26}$. Extraction of total RNA samples from appropriate plant materials and RT-PCR conditions have been described previously ${ }^{27}$. The RNA samples were pretreated extensively with RNasefree DNase to eliminate contaminating genomic DNA before use.

RT-qPCR reactions were conducted in 96-well blocks with an Applied Biosystems 7500 Real-Time PCR System using the SYBR Green I master mix in a volume of $25 \mu \mathrm{l}$. The PCR primers were designed using the Primer Express
}

Software installed into the system and listed in Supplementary Table 1. The twostep thermal cycling profile employed was $15 \mathrm{~s}$ at $94^{\circ} \mathrm{C}$ and $1 \mathrm{~min}$ at $68^{\circ} \mathrm{C}$. An eIF4A gene (At3g13920) was included in the reactions as internal control for normalizing the variations in cDNA amounts used. All RT-qPCR reactions were carried out in biological triplicates using RNA samples extracted from three independent plant materials grown under identical growth conditions. The comparative $\Delta \Delta C_{\mathrm{T}}$ method was employed to evaluate relative quantities of each amplified product in the samples. The threshold cycle $\left(C_{\mathrm{T}}\right)$ was automatically determined for each reaction by the system set with default parameters. The specificity of the PCR reactions was determined by melt curve analysis of the amplified products using the standard method installed in the system.

Measurements of free IAA content. Plants grown on MS-agar plates at $23^{\circ} \mathrm{C}$ for 6 days were incubated at $28^{\circ} \mathrm{C}$ for $24 \mathrm{~h}$. Aerial plants parts were harvested for the extraction of free IAA. Plant materials were finely ground in liquid nitrogen, and $0.1 \mathrm{~g}$ of the powder was extracted in $1 \mathrm{ml}$ of ethyl acetate-formic acid solution (99.5:0.5) containing $20 \mathrm{ng}$ of $\mathrm{D}_{5}$-IAA as a standard. The samples were then vortex mixed for $10 \mathrm{~min}$ and centrifuged at $16,000 \mathrm{~g}$ for $20 \mathrm{~min}$ at $4^{\circ} \mathrm{C}$. The supernatants were then evaporated at room temperature in SpeedVac (Eppendorf, Hamburg, Germany). The pellets were resuspended in $50 \mu \mathrm{l}$ of methanol-water solution $(70: 30)$ and dissolved for 5 min using an ultrasonic bath (Branson, Danbury, CT). IAA content was measured using liquid chromatography (Agilent Technologies, Santa Clara, CA), which was coupled to a mass spectrometer (Applied Biosystems, Foster City, CA), as described previously ${ }^{28}$. Separation was performed on a Zorbax Eclipse column (Agilent technologies). Mobile phases A $(0.05 \%$ acetic acid in water) and B (0.05\% acetic acid in acetonitrile) were used for the separation. The mass spectrometer was operated in the positive ionization mode with an ion spray voltage of $5,500 \mathrm{~V}$ and a turbo gas temperature of $700{ }^{\circ} \mathrm{C}$.

BiFC assay. BiFC assays were carried out as described previously ${ }^{29}$. A full-size FCA gene was fused in-frame to the $3^{\prime}$ end of a DNA sequence encoding the carboxyl-terminal half of yellow fluorescent protein (YFP) in the pSATN-cYFP-C1 vector (E3082). A PIF4 cDNA was fused in-frame to the $3^{\prime}$ end of a DNA sequence encoding the amino-terminal half of YFP in the pSATN-nYFP-C1 vector (E3081). The expression constructs were co-transformed into Arabidopsis protoplasts. The expression of the fusion constructs was visualized by differential interference contrast microscopy and fluorescence microscopy using a Zeiss LSM510 confocal microscope (Carl Zeiss, Yena, Germany). Reconstitution of YFP fluorescence was observed using a confocal microscope with the following YFP filter set up: excitation $515 \mathrm{~nm}, 458 / 514$ dichroic, and emission 560-615 nm BP filter.

Preparation of MBP-FCA fusion protein. The FCA gene was subcloned into the pMAL-c2X Escherichia coli expression vector (NEB, Ipswich, MA) containing a maltose-binding protein (MBP)-coding sequence. Recombinant MBP and MBPFCA fusion proteins were produced in E. coli Rosetta2 (DE3) pLysS strain (Novagen, Madison, WI) and purified as described previously ${ }^{29}$. One-tenth volume of precultured cells $(5 \mathrm{ml})$ was added to $500 \mathrm{ml}$ of Luria-Bertani medium and cultured at $37^{\circ} \mathrm{C}$ until $\mathrm{OD}_{600}=0.3-0.6$. Then, isopropyl- $\beta$-D-thiogalactopyranoside was added to the culture at a final concentration of $0.5 \mathrm{mM}$, and the mixture was incubated for $16 \mathrm{~h}$ at $22^{\circ} \mathrm{C}$ to induce protein production. Cells were harvested and resuspended in $\mathrm{MBP}$ buffer (20 mM Tris- $\mathrm{HCl}, \mathrm{pH}$ 7.4, $200 \mathrm{mM} \mathrm{NaCl}, 1 \mathrm{mM}$ EDTA, $10 \mathrm{mM}$ 2-mercaptoethanol, $1 \mathrm{mM}$ phenylmethylsulfonyl fluoride (PMSF) and protease inhibitor cocktail (Sigma-Aldrich, St Louis, MO; Cat No. P9599)). Cell lysates were prepared by three cycles of freezing and thawing followed by centrifugation. The supernatants were stored at $-80{ }^{\circ} \mathrm{C}$ until use.

In vitro pull-down assay. To examine the interactions of FCA with PIF4, the PIF4 cDNA was amplified by RT-PCR and subcloned into the pGBKT7 vector, which contains the T7 RNA polymerase promoter upstream of a multiple cloning sequence. The $\left[{ }^{35} \mathrm{~S}\right]$ methionine-labelled PIF4 protein was synthesized by in vitro translation using the TNT coupled reticulocyte lysate system (Promega, Madison, $\mathrm{WI})$. Five $\mu \mathrm{l}$ of the ${ }^{35}$ S-labelled protein solution was incubated with $1 \mu \mathrm{g}$ of purified MBP or MBP-FCA fusion protein bound to MBP beads in $1 \mathrm{ml}$ of binding buffer (50 mM Tris-HCl, pH 8.0, $100 \mathrm{mM} \mathrm{NaCl,} \mathrm{10 \%} \mathrm{glycerol,} \mathrm{1 \%} \mathrm{Triton-X-100,} 1 \mathrm{mM}$ PMSF and protease inhibitor cocktail (Sigma-Aldrich)) containing 5\% skim milk for $12 \mathrm{~h}$ at $4^{\circ} \mathrm{C}$. The beads were washed 10 times with TN buffer $(25 \mathrm{mM}$ Tris- $\mathrm{HCl}$, $\mathrm{pH} 8.0,100 \mathrm{mM} \mathrm{NaCl})$. The bound proteins were eluted with $1 \times \mathrm{SDS}$-polyacrylamide gel electrophoresis loading buffer by heating for $10 \mathrm{~min}$ at $70^{\circ} \mathrm{C}$ and subject to SDS-polyacrylamide gel electrophoresis and autoradiography.

ChIP assay. ChIP assays were performed as described previously ${ }^{30}$. The assays were performed in biological triplicates using three independent plant materials grown under identical growth conditions. Plants were grown on MS-agar plates for 6 days and further grown for appropriate time periods at either 23 or $28^{\circ} \mathrm{C}$. Whole plants were harvested for the analysis. Plant materials were vacuum infiltrated with $1 \%(\mathrm{v} / \mathrm{v})$ formaldehyde for cross-linking and ground in liquid nitrogen after quenching the cross-linking process. The ground powder was resuspended in nuclear extraction buffer (1.7 M sucrose, $10 \mathrm{mM}$ Tris-Cl, pH 7.5, $2 \mathrm{mM} \mathrm{MgCl}$, 
0.15\% Triton-X-100, $5 \mathrm{mM} \beta$-mercaptoethanol, $0.1 \mathrm{mM}$ PMSF and protease inhibitor cocktail tablets (Sigma-Aldrich)) and centrifuged at $16,000 \mathrm{~g}$ for $1 \mathrm{~h}$ at $4{ }^{\circ} \mathrm{C}$ to obtain nuclear fraction. The nuclear fraction was lysed with lysis buffer (50 mM Tris-Cl, $\mathrm{pH}$ 8.0, 0.5 M EDTA, $1 \%$ SDS and protease inhibitor cocktail tablets (Sigma-Aldrich)) to extract chromatin. Chromatin preparations were sonicated into $0.4-0.7-\mathrm{kb}$ fragments. Five $\mu \mathrm{g}$ of anti-MYC (Millipore, Billerica, MA; Cat No. 05-724), anti-FLAG (Sigma-Aldrich; Cat No. F1804) or anti-H3K4me2 (Millipore; Cat No. 05-1338) antibody was added to the chromatin solutions, which were precleared with salmon sperm DNA/ Protein G agarose beads (Roche, Indianapolis, IN). The precipitates were eluted from the beads. Cross-links were reversed, and residual proteins were removed by incubation with proteinase $\mathrm{K}$. DNA was recovered using the Promega spin column (Promega). Quantitative PCR was used to determine the amounts of genomic DNA enriched in the chromatin preparations, and the values were normalized to the amount of input in each sample. The primers used are listed in Supplementary Table 1.

Coimmunoprecipitation assay. Coimmunoprecipitation assays were performed as described previously ${ }^{31}$. Plants were grown for 6 days at $23^{\circ} \mathrm{C}$ and further grown for $24 \mathrm{~h}$ at either 23 or $28^{\circ} \mathrm{C}$. Whole plants were harvested for the assays. Plant materials were ground in liquid nitrogen and proteins were extracted in coimmunoprecipitation buffer $(50 \mathrm{mM}$ Tris-Cl pH 7.4, $500 \mathrm{mM} \mathrm{NaCl}, 10 \%$ glycerol, $5 \mathrm{mM}$ EDTA, $1 \%$ Triton-X-100, 1\% Nonidet P-40 and protease inhibitor cocktail tablets (Sigma-Aldrich)). Extract (5\%) was used as the input control. Five $\mu \mathrm{g}$ of anti-FLAG antibody (Sigma-Aldrich; Cat No. F1804) was added to the extract and incubated for $2 \mathrm{~h}$. After the incubation, protein $\mathrm{G}$ agarose beads were added and further incubated for $2 \mathrm{~h}$. The beads were then washed five times with coimmunoprecipitation buffer lacking protease inhibitor cocktail. To elute proteins, $50 \mu \mathrm{l}$ of $2 \times$ SDS loading buffer $(100 \mathrm{mM}$ Tris-Cl pH 6.8, $4 \%$ sodium dodecyl sulfate, $0.2 \%$ bromophenol blue, $20 \%$ glycerol, $200 \mathrm{mM} \mathrm{DTT}$ ) was added to the beads and incubated at $100{ }^{\circ} \mathrm{C}$ for $10 \mathrm{~min}$. Eluted proteins (20\%) was used for IP control. Anti-FLAG antibody (Sigma-Aldrich; Cat No. F1804) and anti-MYC antibody (Millipore; Cat No. 05-724) were used for the detection of FLAG-PIF4 and MYC-FCA, respectively (dilution = 1:4,000). Uncropped blot images of Fig. 4i are shown in Supplementary Fig. 11.

\section{References}

1. Koini, M. A. et al. High temperature-mediated adaptations in plant architecture require the bHLH transcription factor PIF4. Curr. Biol. 19, 408-413 (2009).

2. Crawford, A. J., McLachlan, D. H., Hetherington, A. M. \& Franklin, K. A. High temperature exposure increases plant cooling capacity. Curr. Biol. 22, R396-R397 (2012).

3. Mooney, K. A., Halitschke, R., Kessler, A. \& Agrawal, A. A. Evolutionary tradeoffs in plants mediate the strength of trophic cascades. Science 327, 1642-1644 (2010).

4. Franklin, K. A. et al. PHYTOCHROME-INTERACTING FACTOR 4 (PIF4) regulates auxin biosynthesis at high temperature. Proc. Natl Acad. Sci. USA 108, 20231-20235 (2011)

5. Sun, J., Qi, L., Li, Y., Chu, J. \& Li, C. PIF4-mediated activation of YUCCA8 expression integrates temperature into the auxin pathway in regulating Arabidopsis hypocotyl growth. PLoS Genet. 8, e1002594 (2012).

6. Kumar, S. V. et al. Transcription factor PIF4 controls the thermosensory activation of flowering. Nature 484, 242-245 (2012).

7. Oh, E., Zhu, J. Y. \& Wang, Z. Y. Interaction between BZR1 and PIF4 integrates brassinosteroid and environmental responses. Nat. Cell Biol. 14, 802-809 (2012).

8. Liu, F., Marquardt, S., Lister, C., Swiezewski, S. \& Dean, C. Targeted $3^{\prime}$ processing of antisense transcripts triggers Arabidopsis FLC chromatin silencing. Science 327, 94-97 (2010).

9. Liu, F. et al. The Arabidopsis RNA-binding progtein FCA requires a lysinespecific demethylase 1 homolog to downregulate FLC. Mol. Cell 28, 398-407 (2007)

10. Quesada, V., Macknight, R., Dean, C. \& Simpson, G. G. Autoregulation of FCA pre-mRNA processing controls Arabidopsis flowering time. EMBO J. 22, 3142-3152 (2003).

11. Blázquez, M. A., Ahn, J. H. \& Weigel, D. A thermosensory pathway controlling flowering time in Arabidopsis thaliana. Nat. Genet. 33, 168-171 (2003).

12. Gray, W. M., Östin, A., Sandberg, G., Romano, C. P. \& Estelle, M. High temperature promotes auxin-mediated hypocotyl elongation in Arabidopsis. Proc. Natl Acad. Sci. USA 95, 7197-7202 (1998).

13. Lim, M. H. et al. A new Arabidopsis gene, FLK, encodes an RNA binding protein with $\mathrm{K}$ homology motifs and regulates flowering time via FLOWERING LOCUS C. Plant Cell 16, 731-740 (2004).

14. Yu, C. W. et al. HISTONE DEACETYLASE6 interacts with FLOWERING LOCUS D and regulates flowering in Arabidopsis. Plant Physiol. 156, 173-184 (2011).

15. Choi, K. et al. The FRIGIDA complex activates transcription of FLC, a strong flowering repressor in Arabidopsis, by recruiting chromatin modification factors. Plant Cell 23, 289-303 (2011).
16. Bai, M. Y. et al. Brassinosteroid, gibberellin and phytochrome impinge on a common transcription module in Arabidopsis. Nat. Cell Biol. 14, 810-817 (2012).

17. Muday, G. K., Rahman, A. \& Binder, B. M. Auxin and ethylene: collaborators or competitors? Trends Plant Sci. 17, 181-195 (2012).

18. Fernández-Marcos, M., Sanz, L., Lewis, D. R., Muday, G. K. \& Lorenzo, O. Nitric oxide causes root apical meristem defects and growth inhibition while reducing PIN-FORMED 1 (PIN1)-dependent acropetal auxin transport. Proc. Natl Acad. Sci. USA 108, 18506-18511 (2011).

19. Yoo, S. D., Cho, Y. H. \& Sheen, J. Arabidopsis mesophyll protoplasts: a versatile cell system for transient gene expression analysis. Nat. Protoc. 2, 1565-1572 (2007).

20. Dong, C. H., Agarwal, M., Zhang, Y., Xie, Q. \& Zhu, J. K. The negative regulator of plant cold responses, HOS1, is a RING E3 ligase that mediates the ubiquitination and degradation of ICE1. Proc. Natl Acad. Sci. USA 103, 8281-8286 (2006).

21. Chinnusamy, V. et al. ICE1: a regulator of cold-induced transcriptome and freezing tolerance in Arabidopsis. Genes Dev. 17, 1043-1054 (2003).

22. Gilmour, S. J., Sebolt, A. M., Salazar, M. P., Everard, J. D. \& Thomashow, M. F. Overexpression of the Arabidopsis CBF3 transcriptional activator mimics multiple biochemical changes associated with cold acclimation. Plant Physiol. 124, 1854-1865 (2000).

23. Bäurle, I., Smith, L., Baulcombe, D. C. \& Dean, C. Widespread role for the flowering-time regulators FCA and FPA in RNA-mediated chromatin silencing. Science 318, 109-112 (2007).

24. Jung, J. H., Seo, P. J., Ahn, J. H. \& Park, C. M. Arabidopsis RNA-binding protein FCA regulates microRNA172 processing in thermosensory flowering. J. Biol. Chem. 287, 16007-16016 (2012).

25. Seo, P. J. et al. Cold activation of a plasma membrane-tethered NAC transcription factor induces a pathegen resistance response in Arabidopsis. Plant J. 61, 661-671 (2010).

26. Udvardi, M. K., Czechowski, T. \& Scheible, W. R. Eleven golden rules of quantitative RT-PCR. Plant Cell 20, 1736-1737 (2008).

27. Kim, Y. S. et al. A membrane-bound NAC transcription factor regulates cell division in Arabidopsis. Plant Cell 18, 3132-3144 (2006).

28. Woldemariam, M. G., Onkokesung, N., Baldwin, I. T. \& Galis, I. Jasmonoyl-Lisoleucine hydrolase 1 (JIH1) regulates jasmonoyl-L-isoleucine levels and attenuates plant defenses against herbivores. Plant J. 72, 758-767 (2012).

29. Seo, P. J., Kim, M. J., Ryu, J. Y., Jeong, E. Y. \& Park, C. M. Two splice variants of the IDD14 transcription factor competitively form nonfunctional heterodimers which may regulate starch metabolism. Nat. Commun. 2, 303 (2011).

30. Lee, S., Seo, P. J., Lee, H. J. \& Park, C. M. A NAC transcription factor NTL4 promotes reactive oxygen species production during drought-induced leaf senescence in Arabidopsis. Plant J. 70, 831-844 (2012).

31. Song, Y. H., Smith, R. W., To, B. J., Millar, A. J. \& Imaizumi, T. FKF1 conveys timing information for CONSTANS stabilization in photoperiodic flowering. Science 336, 1045-1049 (2012).

\section{Acknowledgements}

We thank Dr Scott Michaels for providing 35S:FLC transgenic plants. This work was supported by the Leaping Research (20120005600) and Global Research Lab (2012055546) Programs provided by the National Research Foundation of Korea and the Next-Generation BioGreen 21 Program (Plant Molecular Breeding Center No. PJ008103) provided by the Rural Development Administration of Korea. It was also supported in part by the Human Frontier Science Program (RGP0002/2012).

\section{Author contributions}

C.-M.P. conceived and designed the experiments. C.-M.P. prepared the manuscript with contributions from H.-J.L. H.-J.L. examined the effects of ambient temperatures on plant development and gene expression and performed chromatin modification assays. J.-H.J. constructed transgenic plants. L.C.L., S.-G.K. and I.T.B. measured free IAA content. S.L. performed confocal microscopy for BiFC assays. I.T.B. provided scientific discussion on the manuscript.

\section{Additional information}

Supplementary Information accompanies this paper at http://www.nature.com/ naturecommunications

Competing financial interests: The authors declare no competing financial interests.

Reprints and permission information is available online at http://npg.nature.com/ reprintsandpermissions/

How to cite this article: Lee, H.-J. et al. FCA mediates thermal adaptation of stem growth by attenuating auxin action in Arabidopsis. Nat. Commun. 5:5473 doi: 10.1038/ncomms6473 (2014) 